\title{
Quantum teleportation in space and frequency using entangled pairs of photons from a frequency comb
}

\author{
Hongbin Song, ${ }^{1,}{ }^{*}$ Hidehiro Yonezawa, ${ }^{1}$ Katanya B. Kuntz, ${ }^{1}$ Michele Heurs, ${ }^{2}$ and Elanor H. Huntington ${ }^{1}$ \\ ${ }^{1}$ Centre for Quantum Computation and Communication Technology, School of Engineering and Information Technology, \\ University of New South Wales Canberra, ACT 2610, Australia \\ ${ }^{2}$ Albert-Einstein-Institut Hannover, Max-Planck-Institut für Gravitationsphysik and Centre for Quantum Engineering and Space-Time \\ Research (QUEST) Leibniz Universität Hannover, Hannover 30167, Germany
}

(Received 22 August 2014; published 30 October 2014)

\begin{abstract}
Using entangled pairs of photons from a frequency comb and wide-band frequency-resolved homodyne detection, we propose a sequential quantum teleportation protocol for continuous variables that teleports an unknown state in space and frequency. A subthreshold optical parametric oscillator (OPO) produces a comb of entangled pairs of photons separated by the free spectral range of the OPO cavity. Wide-band frequency-resolved homodyne detection enables direct access to the sum and difference of quadratures between different teeth in the comb. Such measurements are Einstein-Podolsky-Rosen nullifiers, and can be used as the basis for teleportation protocols. Our protocol for space-and-frequency teleportation effectively links arbitrary frequency channels for frequencydivision multiplexing, which has applications in universal quantum computation and large-capacity quantum communication.
\end{abstract}

DOI: 10.1103/PhysRevA.90.042337

PACS number(s): 03.67.Hk, 03.67.Mn, 03.67.Ac

\section{INTRODUCTION}

Teleportation is a fundamentally important protocol in quantum information science. For example, teleportation lies at the heart of measurement-based quantum computing (QC), which is a competitive alternative to the traditional circuitbased quantum computing model $[1,2]$. The original proposals for measurement-based QC involved discrete variables (i.e., qubits), but subsequently they have been extended to include continuous-variable (CV) protocols [3,4]. CV quantum information protocols, including measurement-based QC, are of significant interest because of their potential use in large-scale systems where states can be multiplexed across a number of independent modes, including slots in time [5] or frequency [6-9].

Continuous-variable frequency-division-multiplexed (FDM) quantum information, including universal FDM-QC and large-capacity quantum communication [9,10], will rely on the generation of FDM quantum states, such as entangled pairs of photons from a frequency comb (which is defined as a comb of entanglement). A number of combs of entanglement have been experimentally demonstrated, including dozen bipartite entangled states (or dozen squeezing) with a singlefrequency continuously pumped and single-polarization OPO [10], 15 quadripartite cluster states with dual-polarization OPOs [6], 60-mode dual-rail quantum-wire cluster state with dual-frequency continuously pumped and dual-polarization OPOs [7], or ten-partite spectral entangled state with OPOs that are synchronously pumped by femtosecond pulse trains [9].

While generating combs of entanglement is important, it is also important to develop methods for manipulations and interactions among frequency modes in a comb. For this purpose, teleportation in frequency space will be a key protocol, where an unknown state in a certain frequency mode

\footnotetext{
*hongbin.song@yahoo.com.au
}

is teleported to that in another frequency mode via a comb of entanglement. Different frequency modes can effectively interact by teleporting a state to a different frequency followed by frequency degenerate interactions, or unitary operations can be realized through a sequence of teleportation in a measurement-based fashion.

In this paper, we develop a space-and-frequency teleportation protocol with simple resource states and single homodyne detection. In our methods, we use continuous-wave (cw) multiple bipartite entangled states as in Ref. [10] rather than multipartite entangled states [6-9]. We utilize wide-band frequency-resolved homodyne detection, which enables us to access each of the teeth in a comb of entanglement. Electronic demodulation following wide-band homodyne detection facilitates direct access to the sum and difference of quadratures between different sideband modes [11], which implies that the Einstein-Podolsky-Rosen (EPR) nullifiers required for effective teleportation [7,12] are simultaneously produced during the measurement process. Based on these insights, we propose a teleportation protocol that can teleport an unknown state to a different frequency and physical location so that the frequency channels in FDM can effectively interact. In addition, we extend this teleportation protocol to sequential teleportation, which performs a series of teleportation at once via single wide-band homodyne detection. This has the potential for more flexible transformations to be implemented, which could be used in new efficient measurement-based QC [5]. The methods we proposed contribute to the set of FDM-based techniques that are essential for large-scale quantum information protocols to become a practical reality.

This paper is structured as follows. In Sec. II, we present an approach to measuring pairs of sidebands in a comb of entanglement. We discuss a protocol for entanglement swapping between the teeth in the comb in Sec. III, and propose a protocol for quantum teleportation based on the frequency comb in Sec. IV. Then we extend the protocol to sequential teleportation in Sec. V. A summary is presented in Sec. VI. 


\section{MEASUREMENT OF SIDEBANDS IN A COMB OF ENTANGLEMENT}

Degenerate OPOs are generally used to produce CV squeezed vacuum states. It is known that the output from an OPO can be a multimode squeezed vacuum state with EPRlike correlations between pairs of nondegenerate frequency modes $[13,14]$. A comb of entanglement generated from a subthreshold, degenerate OPO pumped by a cw laser has been experimentally demonstrated using wide-band homodyne detectors [10]. Within the phase-matching bandwidth of the nonlinear crystal in the OPO, a pump photon with a frequency of $2 \omega_{0}$ is converted to photon pairs whose frequencies are symmetrically distributed around the degenerate frequency, $\omega_{0}$. A wide-band homodyne detector followed by electronic demodulation enables us to access each pair of sideband modes [11].

We define quadratures for each frequency sideband mode as [14],

$$
\begin{aligned}
& \widetilde{X}(\omega)=\widetilde{A}(\omega)^{\dagger}+\widetilde{A}(\omega), \\
& \widetilde{Y}(\omega)=i\left[\widetilde{A}(\omega)^{\dagger}-\widetilde{A}(\omega)\right],
\end{aligned}
$$

where $\widetilde{A}(\omega)$ is a Fourier transform of an annihilation operator $A(t)$. Note that these definitions are different from common definitions of $\widetilde{X}^{\prime}(\omega)=\widetilde{A}(-\omega)^{\dagger}+\widetilde{A}(\omega)$ and $\widetilde{Y}^{\prime}(\omega)=$ $i\left[\widetilde{A}(-\omega)^{\dagger}-\widetilde{A}(\omega)\right]$. The OPO output shows EPR-like quantum correlations between the upper and lower sidebands. This can be written as [14],

$$
\begin{aligned}
& \left\langle\left[\delta \widetilde{Y}_{\mathrm{opo}}\left(\omega_{0}+k \Omega+\omega^{\prime}\right)+\delta \widetilde{Y}_{\mathrm{opo}}\left(\omega_{0}-k \Omega-\omega^{\prime}\right)\right]^{2}\right\rangle \\
& \quad=\left\langle\left[\delta \widetilde{X}_{\mathrm{opo}}\left(\omega_{0}+k \Omega+\omega^{\prime}\right)-\delta \widetilde{X}_{\mathrm{opo}}\left(\omega_{0}-k \Omega-\omega^{\prime}\right)\right]^{2}\right\rangle \\
& \quad=2 e^{-2 r}<2 \quad\left(\frac{-\Delta \omega_{B}}{2}<\omega^{\prime}<\frac{+\Delta \omega_{B}}{2}\right),
\end{aligned}
$$

where $\Omega$ is the free spectral range (FSR) of the OPO cavity, $\Delta \omega_{B}$ is the linewidth of the OPO cavity, which roughly corresponds to the bandwidth of the squeezing at each cavity resonance, $r>0$ is the squeezing parameter, and $k$ is an integer representing the order of the FSR of interest. Here we assume the phase-matching bandwidth of the OPO is much broader than the bandwidth of a homodyne detector, and we shall therefore neglect it.

The teeth in the comb can be accessed with wide-band homodyne detection followed by frequency demodulation as shown in Fig. 1(a). In particular, this measurement technique produces EPR nullifiers of sideband modes that are equivalent to optical mixing followed by measurements on two spatially separated modes, as shown in Fig. 1(b). This is a key insight for the protocols we propose later.

First, let us consider the output of homodyne detection. In the homodyne detection, we combine an input $A_{s}(t)$ with a local oscillator (LO) $A_{L O}(t)$ on a 50:50 beam splitter as,

$$
\begin{aligned}
& A_{r_{s}}(t)=\frac{A_{s}(t)+e^{i \theta_{L O}} A_{L O}(t)}{\sqrt{2}}, \\
& A_{t_{s}}(t)=\frac{A_{s}(t)-e^{i \theta_{L O}} A_{L O}(t)}{\sqrt{2}},
\end{aligned}
$$

where $\theta_{L O}$ is the relative phase between the $\mathrm{LO}$ and the input. We measure both modes $A_{r_{s}}(t)$ and $A_{t_{s}}(t)$ by photodetectors and subtract photocurrents as,

$$
I(t)=G_{\operatorname{det}}\left[A_{r_{s}}^{\dagger}(t) A_{r_{s}}(t)-A_{t_{s}}^{\dagger}(t) A_{t_{s}}(t)\right],
$$

where $G_{\text {det }}$ represents the transimpedance gains of the photodetectors. Here we define that $A_{L O}(t)=\left[\bar{A}_{L O}+\right.$ $\left.\delta A_{L O}(t)\right] e^{i \omega_{L O} t}$ with the LO angular frequency $\omega_{L O}$, and $A_{s}(t)=\bar{A}_{s}+\delta A_{s}(t)$. In addition, we assume that $\bar{A}_{L O}^{*}=$ $\bar{A}_{L O}, \bar{A}_{s}^{*}=\bar{A}_{s}$, and $\bar{A}_{L O} \gg \bar{A}_{s}$. Thus, we have the following expression for the fluctuation of the homodyne output,

$$
\delta I(t)=\bar{A}_{L O} G_{\mathrm{det}}\left[e^{-i \theta_{L O}} e^{-i \omega_{L O} t} \delta A_{s}(t)+e^{i \theta_{L O}} e^{i \omega_{L O} t} \delta A_{s}^{\dagger}(t)\right]
$$

In particular, if we adjust the LO phase to be $\theta_{L O}=\frac{\pi}{2}$, we obtain,

$$
\delta I(t)=i \bar{A}_{L O} G_{\mathrm{det}}\left[e^{i \omega_{L O} t} \delta A_{s}^{\dagger}(t)-e^{-i \omega_{L O} t} \delta A_{s}(t)\right] .
$$

In our setup, we demodulate this signal $\delta I(t)$ with cosine and sine functions. We duplicate the signal $\delta I(t)$, then multiply $\cos \left(\omega_{m} t\right)$ and $\sin \left(\omega_{m} t\right)$, respectively. The results are,

$$
\begin{aligned}
\delta I_{\mathrm{cosm}}(t)= & \frac{i \bar{A}_{L O} G_{\mathrm{det}}}{2}\left[e^{i\left(\omega_{L O}+\omega_{m}\right) t} \delta A_{S}^{\dagger}(t)+e^{i\left(\omega_{L O}-\omega_{m}\right) t} \delta A_{S}^{\dagger}(t)\right. \\
& \left.-e^{-i\left(\omega_{L O}+\omega_{m}\right) t} \delta A_{S}(t)-e^{-i\left(\omega_{L O}-\omega_{m}\right) t} \delta A_{S}(t)\right], \quad(8) \\
\delta I_{\mathrm{sinm}}(t)= & \frac{\bar{A}_{L O} G_{\mathrm{det}}}{2}\left[e^{i\left(\omega_{L O}+\omega_{m}\right) t} \delta A_{S}^{\dagger}(t)-e^{i\left(\omega_{L O}-\omega_{m}\right) t} \delta A_{S}^{\dagger}(t)\right. \\
& \left.+e^{-i\left(\omega_{L O}+\omega_{m}\right) t} \delta A_{S}(t)-e^{-i\left(\omega_{L O}-\omega_{m}\right) t} \delta A_{S}(t)\right] .
\end{aligned}
$$

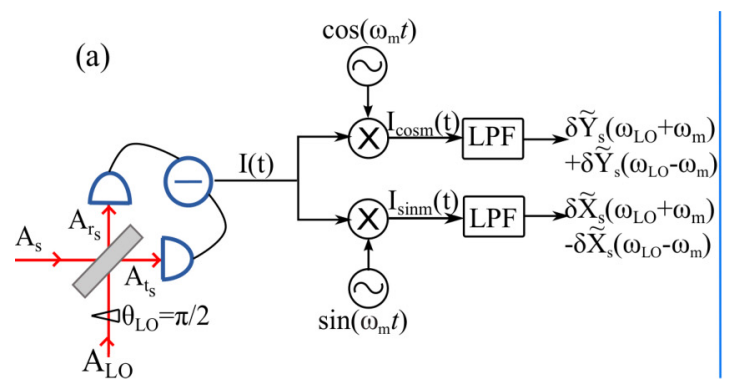

(b)

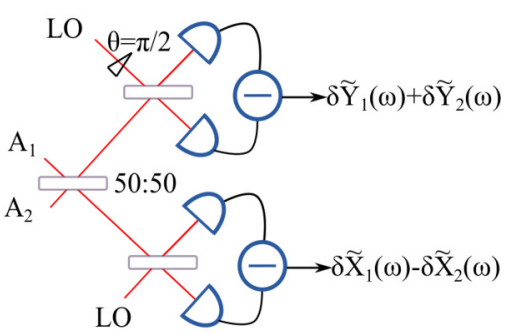

FIG. 1. (Color online) (a) Schematic diagram of wide-band frequency-resolved homodyne measurement on two teeth in a frequency comb, $A_{s}$. (b) Equivalent optical mixing and measurements on two spatially separated modes, $A_{1}$ and $A_{2}$. LPF: low-pass filter. 

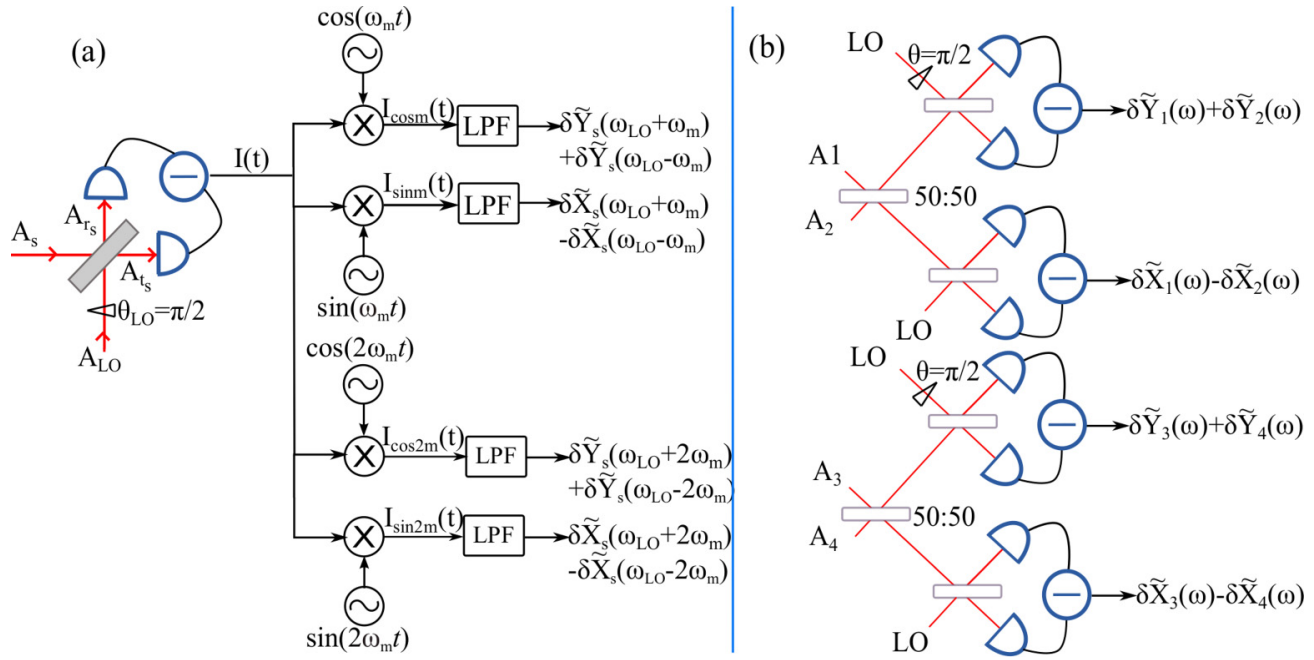

FIG. 2. (Color online) (a) Schematic diagram of wide-band frequency-resolved homodyne measurement on four teeth in a frequency comb, $A_{s}$. By extending the demodulation with signals of different frequencies, we can simultaneously access each tooth in the comb. (b) Equivalent optical mixing and measurements on four spatially separated modes, $A_{1}, A_{2}, A_{3}$, and $A_{4}$.

The Fourier transform of these two signals can be written with sideband quadratures defined in Eq. (1) as,

$$
\begin{aligned}
\delta \widetilde{I}_{\operatorname{cosm}}(\omega)= & \frac{i \bar{A}_{L O} G_{\mathrm{det}}}{4}\left[\delta \widetilde{X}_{s}\left(\omega_{L O}+\omega_{m}-\omega\right)\right. \\
& -i \delta \widetilde{Y}_{s}\left(\omega_{L O}+\omega_{m}-\omega\right) \\
& +\delta \widetilde{X}_{S}\left(\omega_{L O}-\omega_{m}-\omega\right)-i \delta \widetilde{Y}_{s}\left(\omega_{L O}-\omega_{m}-\omega\right) \\
& -\delta \widetilde{X}_{s}\left(\omega_{L O}+\omega_{m}+\omega\right)-i \delta \widetilde{Y}_{s}\left(\omega_{L O}+\omega_{m}+\omega\right) \\
& \left.-\delta \widetilde{X}_{S}\left(\omega_{L O}-\omega_{m}+\omega\right)-i \delta \widetilde{Y}_{s}\left(\omega_{L O}-\omega_{m}+\omega\right)\right],
\end{aligned}
$$

$$
\begin{aligned}
\delta \widetilde{I}_{\text {sinm }}(\omega)= & \frac{\bar{A}_{L O} G_{\mathrm{det}}}{4}\left[\delta \widetilde{X}_{S}\left(\omega_{L O}+\omega_{m}-\omega\right)\right. \\
& -i \delta \widetilde{Y}_{s}\left(\omega_{L O}+\omega_{m}-\omega\right) \\
& -\delta \widetilde{X}_{s}\left(\omega_{L O}-\omega_{m}-\omega\right)+i \delta \tilde{Y}_{s}\left(\omega_{L O}-\omega_{m}-\omega\right) \\
& +\delta \widetilde{X}_{S}\left(\omega_{L O}+\omega_{m}+\omega\right)+i \delta \widetilde{Y}_{s}\left(\omega_{L O}+\omega_{m}+\omega\right) \\
& \left.-\delta \widetilde{X}_{S}\left(\omega_{L O}-\omega_{m}+\omega\right)-i \delta \tilde{Y}_{s}\left(\omega_{L O}-\omega_{m}+\omega\right)\right]
\end{aligned}
$$

Note that the Fourier transform of $A_{s}^{\dagger}(t)$ is $A_{s}(-\omega)^{\dagger}$. Those expressions of Eq. (10) and Eq. (11) are rather complex. However, if we focus on the DC terms $(\omega=0)$, the physical meaning is clearer,

$$
\begin{aligned}
& \delta \widetilde{I}_{\operatorname{cosm}}(\omega=0) \\
& \quad=\frac{\bar{A}_{L O} G_{\mathrm{det}}}{2}\left[\delta \widetilde{Y}_{S}\left(\omega_{L O}+\omega_{m}\right)+\delta \widetilde{Y}_{s}\left(\omega_{L O}-\omega_{m}\right)\right], \\
& \delta \widetilde{I}_{\operatorname{sinm}}(\omega=0) \\
& \quad=\frac{\bar{A}_{L O} G_{\mathrm{det}}}{2}\left[\delta \widetilde{X}_{S}\left(\omega_{L O}+\omega_{m}\right)-\delta \widetilde{X}_{S}\left(\omega_{L O}-\omega_{m}\right)\right] .
\end{aligned}
$$

These indicate that we can directly access the sum of phase quadratures and difference of amplitude quadratures between frequency sidebands symmetrically around $\omega_{L O}$ by simple measurements using wide-band frequency-resolved homodyne detection with a constant LO phase. That is, the EPR nullifiers are generated through the measurement without using optical mixing as shown in Fig. 1(b) $[5,15]$. It is noteworthy to mention that this still obeys the Heisenberg uncertainty principle, since the sum and difference of quadratures, $\delta \widetilde{Y}_{s}\left(\omega_{L O}+\omega_{m}\right)+\delta \widetilde{Y}_{s}\left(\omega_{L O}-\omega_{m}\right)$ and $\delta \widetilde{X}_{s}\left(\omega_{L O}+\omega_{m}\right)-\delta \widetilde{X}_{s}\left(\omega_{L O}-\omega_{m}\right)$, commute.

As can be seen in Eq. (12) and Eq. (13), we can adjust the LO frequency $\omega_{L O}$ and the demodulation frequency $\omega_{m}$ to measure a specific pair of sidebands. For instance, we can measure the correlation shown in Eq. (2) between the sideband pair of $\omega_{0}-\Omega$ and $\omega_{0}+\Omega$ from the OPO by simply setting $\omega_{L O}=\omega_{0}$ and $\omega_{m}=\Omega$.

The significance of this method is demonstrated by how easily it can be extended to a number of sidebands within the detection bandwidth. As an example, measurement of four teeth in a comb is shown in Fig. 2(a). In this setting, the homodyne outputs are demodulated with $\cos \left(\omega_{m} t\right), \sin \left(\omega_{m} t\right)$, $\cos \left(2 \omega_{m} t\right)$, and $\sin \left(2 \omega_{m} t\right)$, respectively. We can access four combinations of quadratures as shown in Fig. 2(a). That is, this measurement technique produces a number of EPR nullifiers simultaneously by just one homodyne detection [Fig. 2(b) shows an example of four modes]. Thus this approach has great potential to reduce multiple beam splitter transformations followed by multiple homodyne detectors to just one homodyne detector for suitable quantum information protocols.

\section{ENTANGLEMENT SWAPPING}

From Sec. II, we see that we can access the EPR nullifiers for arbitrary pairs of teeth in the comb of entanglement by varying the LO and demodulation frequencies. In this section, we demonstrate how to put those measurement techniques to use. Here we shall describe an entanglement swapping protocol that creates entanglement between $\omega_{0}-3 \Omega$ and $\omega_{0}+$ $\Omega$ sidebands from two pairs of entanglement, an entangled pair of $\omega_{0} \pm \Omega$ sidebands and another entangled pair of $\omega_{0} \pm 3 \Omega$ 


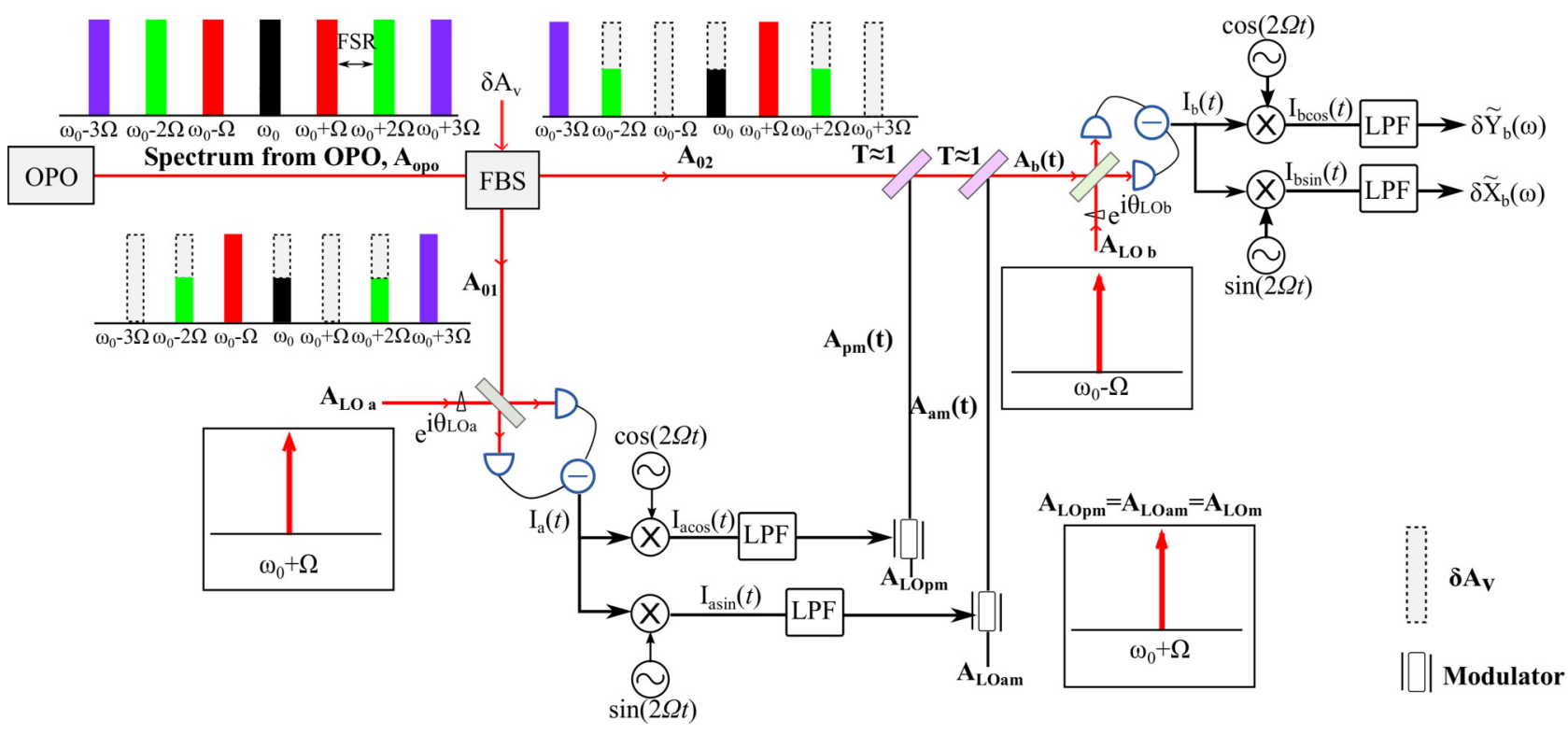

FIG. 3. (Color online) Schematic diagram of entanglement swapping.

sidebands. A schematic diagram of the protocol is shown in Fig. 3. The frequency-dependent beam splitter (FBS) is an unbalanced Mach-Zehnder interferometer (UMZI) [16] with outputs,

$$
\begin{aligned}
& \delta \widetilde{A}_{01}(\omega)=\frac{1}{2}\left[\delta \widetilde{A}_{\mathrm{opo}}(\omega)\left(1+e^{i \omega \tau}\right)+\delta \widetilde{A}_{v}(\omega)\left(e^{i \omega \tau}-1\right)\right] \\
& \delta \widetilde{A}_{02}(\omega)=\frac{1}{2}\left[\delta \widetilde{A}_{\mathrm{opo}}(\omega)\left(e^{i \omega \tau}-1\right)+\delta \widetilde{A}_{v}(\omega)\left(e^{i \omega \tau}+1\right)\right]
\end{aligned}
$$

where $\tau$ is the time delay introduced by the length difference between the two unbalanced arms of the UMZI. When $\omega_{0} \tau=$ $2 m \pi+\frac{\pi}{2}(m=0,1,2, \ldots)$ and $\Omega \tau=\frac{\pi}{2}$, the FBS separates the sideband pair $\omega_{0}-\Omega$ and $\omega_{0}+3 \Omega$ from the pair of $\omega_{0}+\Omega$ and $\omega_{0}-3 \Omega$. The even-order teeth in the comb are contaminated by the vacuum noise, $\delta A_{v}$, and remain on both outputs, $A_{01}$ and $A_{02}$.

First we measure one of the output of the FBS, $A_{01}$. Here we use an LO of $A_{L O_{a}}(t)=\left[\bar{A}_{L O_{a}}+\delta A_{L O_{a}}(t)\right] e^{i \omega_{L O_{a}} t}$ with $\omega_{L O_{a}}=\omega_{0}+\Omega$. In addition, we set the LO phase as $\theta_{L O_{a}}=\frac{\pi}{2}$ as in the previous section, and set the demodulation frequency as $\omega_{m_{a}}=2 \Omega$.

According to Eqs. (8) and (9), we have

$$
\begin{aligned}
\delta I_{a_{\mathrm{cos}}}(t) & \\
= & \frac{i \bar{A}_{L O_{a}} G_{\mathrm{det}}}{2}\left[-e^{-i\left(\omega_{0}-\Omega\right) t} \delta A_{01}(t)-e^{-i\left(\omega_{0}+3 \Omega\right) t} \delta A_{01}(t)\right. \\
& \left.+e^{i\left(\omega_{0}+3 \Omega\right) t} \delta A_{01}^{\dagger}(t)+e^{i\left(\omega_{0}-\Omega\right) t} \delta A_{01}^{\dagger}(t)\right] \\
\delta I_{a_{\mathrm{sin}}}(t) & \\
= & \frac{\bar{A}_{L O_{a}} G_{\mathrm{det}}}{2}\left[-e^{-i\left(\omega_{0}-\Omega\right) t} \delta A_{01}(t)+e^{-i\left(\omega_{0}+3 \Omega\right) t} \delta A_{01}(t)\right. \\
& \left.+e^{i\left(\omega_{0}+3 \Omega\right) t} \delta A_{01}^{\dagger}(t)-e^{i\left(\omega_{0}-\Omega\right) t} \delta A_{01}^{\dagger}(t)\right] .
\end{aligned}
$$

Let us use those measurement results to displace the other output of the FBS, $A_{02}$, so as to achieve entanglement swapping. We implement amplitude and phase modulation with $I_{\text {asin }}(t)$ and $I_{\text {acos }}(t)$ on frequency-shifted local oscillators, which can be expressed respectively as

$$
\begin{aligned}
& A_{a m}(t)=A_{L O_{a m}}(t)\left[1+G_{a m} I_{\mathrm{asin}}(t)\right], \\
& A_{p m}(t)=A_{L O_{p m}}(t)\left[1+i G_{p m} I_{\mathrm{acos}}(t)\right],
\end{aligned}
$$

where $A_{L O_{a m}}(t)=\left[\bar{A}_{L O_{m}}+\delta A_{L O_{a m}}(t)\right] e^{i\left(\omega_{0}+\Omega\right) t}, A_{L O_{p m}}(t)=$ $\left[\bar{A}_{L O_{m}}+\delta A_{L O_{p m}}(t)\right] e^{i\left(\omega_{0}+\Omega\right) t}$, and $G_{a m}$ and $G_{p m}$ are the electro-to-optical gains of the modulators. For simplicity, we set $G_{a m}=G_{p m}=G_{m}$, and then we obtain,

$$
\begin{aligned}
\delta A_{a m}(t)= & \bar{A}_{L O_{m}} G_{m} e^{i\left(\omega_{0}+\Omega\right) t} \delta I_{\mathrm{asin}}(t) \\
= & g_{a m}\left[\left(-e^{2 i \Omega t}+e^{-2 i \Omega t}\right) \delta A_{01}(t)\right. \\
& \left.+\left(e^{i\left(2 \omega_{0}+4 \Omega\right) t}-e^{2 i \omega_{0} t}\right) \delta A_{01}^{\dagger}(t)\right], \\
\delta A_{p m}(t)= & i \bar{A}_{L O_{m}} G_{m} e^{i\left(\omega_{0}+\Omega\right) t} \delta I_{\mathrm{acos}}(t) \\
= & g_{p m}\left[\left(e^{2 i \Omega t}+e^{-2 i \Omega t}\right) \delta A_{01}(t)\right. \\
& \left.-\left(e^{i\left(2 \omega_{0}+4 \Omega\right) t}+e^{2 i \omega_{0} t}\right) \delta A_{01}^{\dagger}(t)\right],
\end{aligned}
$$

where $g_{a m}=g_{p m}=\frac{\bar{A}_{L O m} \bar{A}_{L O_{a}} G_{m} G_{\mathrm{det}}}{2}$ with $g_{a m}$ and $g_{p m}$ being the gain of amplitude modulation and phase modulation, respectively. Correspondingly, in the frequency domain, we have

$$
\begin{aligned}
\delta \widetilde{A}_{a m}(\omega)= & g_{a m}\left[-\delta \widetilde{A}_{01}(\omega-2 \Omega)+\delta \widetilde{A}_{01}(\omega+2 \Omega)\right. \\
& \left.+\delta \widetilde{A}_{01}\left(2 \omega_{0}+4 \Omega-\omega\right)^{\dagger}-\delta \widetilde{A}_{01}\left(2 \omega_{0}-\omega\right)^{\dagger}\right], \\
\delta \widetilde{A}_{p m}(\omega)= & g_{p m}\left[\delta \widetilde{A}_{01}(\omega-2 \Omega)+\delta \widetilde{A}_{01}(\omega+2 \Omega)\right. \\
& \left.-\delta \widetilde{A}_{01}\left(2 \omega_{0}+4 \Omega-\omega\right)^{\dagger}-\delta \widetilde{A}_{01}\left(2 \omega_{0}-\omega\right)^{\dagger}\right] .
\end{aligned}
$$

Then we displace $A_{02}$ with these amplitude- and phasemodulated signals through mirrors with approximately unit transmission (i.e., $T \approx 1$ ). We have

$$
A_{b}(t)=\left[T A_{02}(t)+R A_{p m}(t)\right] T+R A_{a m}(t) .
$$


Thus, in the frequency domain,

$$
\delta \widetilde{A}_{b}(\omega)=T^{2} \delta \widetilde{A}_{02}(\omega)+T R \delta \widetilde{A}_{p m}(\omega)+R \delta \widetilde{A}_{a m}(\omega) .
$$

Substituting Eq. (20) into (22) and setting $T^{2}=4 R T g_{p m}=$ $4 R g_{a m} \simeq 1$, we have

$$
\delta \widetilde{A}_{b}(\omega)=\delta \widetilde{A}_{02}(\omega)+\frac{1}{2}\left[\delta \widetilde{A}_{01}(\omega+2 \Omega)-\delta \widetilde{A}_{01}\left(2 \omega_{0}-\omega\right)^{\dagger}\right] .
$$

Therefore,

$$
\begin{aligned}
\delta \widetilde{X}_{b}(\omega) & =\delta \widetilde{X}_{02}(\omega)+\frac{1}{2}\left[\delta \widetilde{X}_{01}(\omega+2 \Omega)-\delta \widetilde{X}_{01}\left(2 \omega_{0}-\omega\right)\right] \\
\delta \widetilde{Y}_{b}(\omega) & =\delta \widetilde{Y}_{02}(\omega)+\frac{1}{2}\left[\delta \widetilde{Y}_{01}(\omega+2 \Omega)+\delta \widetilde{Y}_{01}\left(2 \omega_{0}-\omega\right)\right] .
\end{aligned}
$$

Equation (24) describes the output of the frequency entanglement swapping protocol. If the protocol works, there should now be entanglement between the $\omega_{0}-3 \Omega$ and $\omega_{0}+\Omega$ sidebands where previously there had been entanglement between $\omega_{0} \pm \Omega$ sidebands and, independently, entanglement between $\omega_{0} \pm 3 \Omega$ sidebands. In order to verify entanglement between $\omega_{0}-3 \Omega$ and $\omega_{0}+\Omega$ sidebands, we measure $\delta A_{b}$ with LO frequency $\omega_{L O_{b}}=\omega_{0}-\Omega$, LO phase $\theta_{L O_{b}}=\frac{\pi}{2}$ and demodulation frequency $\omega_{m_{b}}=2 \Omega$. Using Eq. (12) and Eq. (13) we obtain,

$$
\begin{aligned}
& \delta \widetilde{I}_{b_{\text {cos }}}(\omega=0)=\frac{\bar{A}_{L O_{b}} G_{\text {det }}}{2}\left[\delta \widetilde{Y}_{b}\left(\omega_{0}+\Omega\right)+\delta \widetilde{Y}_{b}\left(\omega_{0}-3 \Omega\right)\right], \\
& \delta \widetilde{I}_{b_{\text {sin }}}(\omega=0)=\frac{\bar{A}_{L O_{b}} G_{\text {det }}}{2}\left[\delta \widetilde{X}_{b}\left(\omega_{0}+\Omega\right)-\delta \widetilde{X}_{b}\left(\omega_{0}-3 \Omega\right)\right] .
\end{aligned}
$$

Substituting Eq. (24) into Eq. (25), and using quadratures of the OPO, $\delta \widetilde{X}_{\mathrm{opo}}(\omega)$ and $\delta \widetilde{Y}_{\mathrm{opo}}(\omega)$, we have

$$
\begin{aligned}
\delta \widetilde{I}_{b_{\mathrm{cos}}}(\omega=0)= & \frac{\bar{A}_{L O_{b}} G_{\mathrm{det}}}{2}\left[\delta \widetilde{Y}_{\mathrm{opo}}\left(\omega_{0}+\Omega\right)+\delta \widetilde{Y}_{\mathrm{opo}}\left(\omega_{0}-\Omega\right)\right. \\
& \left.+\delta \widetilde{Y}_{\mathrm{opo}}\left(\omega_{0}+3 \Omega\right)+\delta \widetilde{Y}_{\mathrm{opo}}\left(\omega_{0}-3 \Omega\right)\right], \\
\delta \widetilde{I}_{b_{\mathrm{sin}}}(\omega=0)= & \frac{\bar{A}_{L O_{b}} G_{\mathrm{det}}}{2}\left[\delta \widetilde{X}_{\mathrm{opo}}\left(\omega_{0}+\Omega\right)-\delta \widetilde{X}_{\mathrm{opo}}\left(\omega_{0}-\Omega\right)\right. \\
& \left.+\delta \widetilde{X}_{\mathrm{opo}}\left(\omega_{0}+3 \Omega\right)-\delta \widetilde{X}_{\mathrm{opo}}\left(\omega_{0}-3 \Omega\right)\right] .
\end{aligned}
$$

According to the EPR correlation relationship between sidebands of the OPO shown in Eq. (2), we can calculate variances as,

$$
\begin{aligned}
& \left\langle\left[\delta \widetilde{I}_{b_{\mathrm{cos}}}(\omega=0)\right]^{2}\right\rangle /\left(\frac{\bar{A}_{L O_{b}} G_{\mathrm{det}}}{2}\right)^{2} \\
& =\left\langle\left[\delta \widetilde{Y}_{\mathrm{opo}}\left(\omega_{0}+\Omega\right)+\delta \widetilde{Y}_{\mathrm{opo}}\left(\omega_{0}-\Omega\right)\right.\right. \\
& \left.\left.\quad+\delta \widetilde{Y}_{\mathrm{opo}}\left(\omega_{0}+3 \Omega\right)+\delta \widetilde{Y}_{\mathrm{opo}}\left(\omega_{0}-3 \Omega\right)\right]^{2}\right\rangle=4 e^{-2 r},
\end{aligned}
$$

$$
\begin{aligned}
\langle[ & \left.\left.\delta \widetilde{I}_{b_{\mathrm{sin}}}(\omega=0)\right]^{2}\right\rangle /\left(\frac{\bar{A}_{L O_{b}} G_{\mathrm{det}}}{2}\right)^{2} \\
= & \left\langle\left[\delta \widetilde{X}_{\mathrm{opo}}\left(\omega_{0}+\Omega\right)-\delta \widetilde{X}_{\mathrm{opo}}\left(\omega_{0}-\Omega\right)\right.\right. \\
& \left.\left.+\delta \widetilde{X}_{\mathrm{opo}}\left(\omega_{0}+3 \Omega\right)-\delta \widetilde{X}_{\mathrm{opo}}\left(\omega_{0}-3 \Omega\right)\right]^{2}\right\rangle \\
= & 4 e^{-2 r} .
\end{aligned}
$$

If we measure a vacuum mode, that is when the mode $b$ in Eq. (25) is a vacuum mode, we will have $\left\langle\left[\delta \widetilde{I}_{b_{\text {cos }}}(\omega=\right.\right.$ $\left.0)]^{2}\right\rangle=\left(\frac{\bar{A}_{L O_{b}} G_{\mathrm{det}}}{2}\right)^{2} \times 2$ and $\left\langle\left[\delta \tilde{I}_{b_{\text {sin }}}(\omega=0)\right]^{2}\right\rangle=\left(\frac{\bar{A}_{L O_{b}} G_{\mathrm{det}}}{2}\right)^{2} \times$ 2. In other words, the right-hand sides of Eq. $(27)$ will be 2 in the case of a vacuum mode. Thus, if $4 e^{-2 r}<2$ (that is, the initial entanglement is larger than $3 \mathrm{~dB}$ ), we obtain entanglement between $\omega_{0}-3 \Omega$ and $\omega_{0}+\Omega$ sidebands in the output mode $b$, and accomplish entanglement swapping. Note that this $3 \mathrm{~dB}$ requirement of entanglement is intrinsic for entanglement swapping protocols [17].

This analysis shows how entanglement swapping in frequency space can be implemented using two EPR sideband pairs. It is straightforward to apply this protocol to other frequency modes by using other EPR sideband pairs. This method enables manipulation of frequency channels in FDM, and can be an essential protocol in FDM. What is more interesting, however, will be the method to exploit many pairs of EPR entanglement simultaneously rather than using just two pairs of EPR entanglement. In the following section, we shall show how multiple pairs of entanglement in a comb can be put to use as a resource for sequential quantum teleportation.

\section{QUANTUM TELEPORTATION USING A COMB OF ENTANGLEMENT}

In this section, we present quantum teleportation using a pair of entangled sidebands, and then in the next section we will extend it to sequential quantum teleportation. Figure 4 shows the schematic of quantum teleportation, where we use only the first pair of teeth in a comb of entanglement at $\omega_{0}-\Omega$ and $\omega_{0}+\Omega$. We will teleport an input mode at $\omega_{0}$ to a spatially separated mode at $\omega_{0}+\Omega$. Different from the protocol discussed in the previous section, the frequency beam splitters shown in this protocol, being FBS1 and FBS2, are resonant cavities with $\Omega_{F B S_{1,2}} \gg \Omega$ and $\Delta \omega_{B}<\Delta \omega_{F B S_{1,2}}<$ $\Omega$, where $\Omega_{F B S_{1,2}}$ and $\triangle \omega_{F B S_{1,2}}$ are the FSRs and linewidths of FBS1 and FBS2. The resonant frequency of FBS1 is tuned to $\omega_{0}+\Omega$ so that it spatially separates the tooth at $\omega_{0}+\Omega$ from the remaining teeth $\omega_{0}-\Omega$ and $\omega_{0}$, as shown in Fig. 4 . We have outputs of FBS1 at $A_{01}$ and $A_{02}$ in the frequency domain as

$$
\begin{aligned}
& \widetilde{A}_{01}(\omega)= \begin{cases}\delta \widetilde{A}_{v}(\omega) & \omega_{0}+\Omega-\frac{\Delta \omega_{F B S_{1}}}{2}<\omega<\omega_{0}+\Omega+\frac{\Delta \omega_{F B S_{1}}}{2} \\
\widetilde{A}_{\mathrm{opo}}(\omega) & \text { others. }\end{cases} \\
& \widetilde{A}_{02}(\omega)= \begin{cases}\widetilde{A}_{\mathrm{opo}}(\omega) & \omega_{0}+\Omega-\frac{\Delta \omega_{F B S_{1}}}{2}<\omega<\omega_{0}+\Omega+\frac{\Delta \omega_{F B S_{1}}}{2} \\
\delta \widetilde{A}_{v}(\omega) & \text { others. }\end{cases}
\end{aligned}
$$




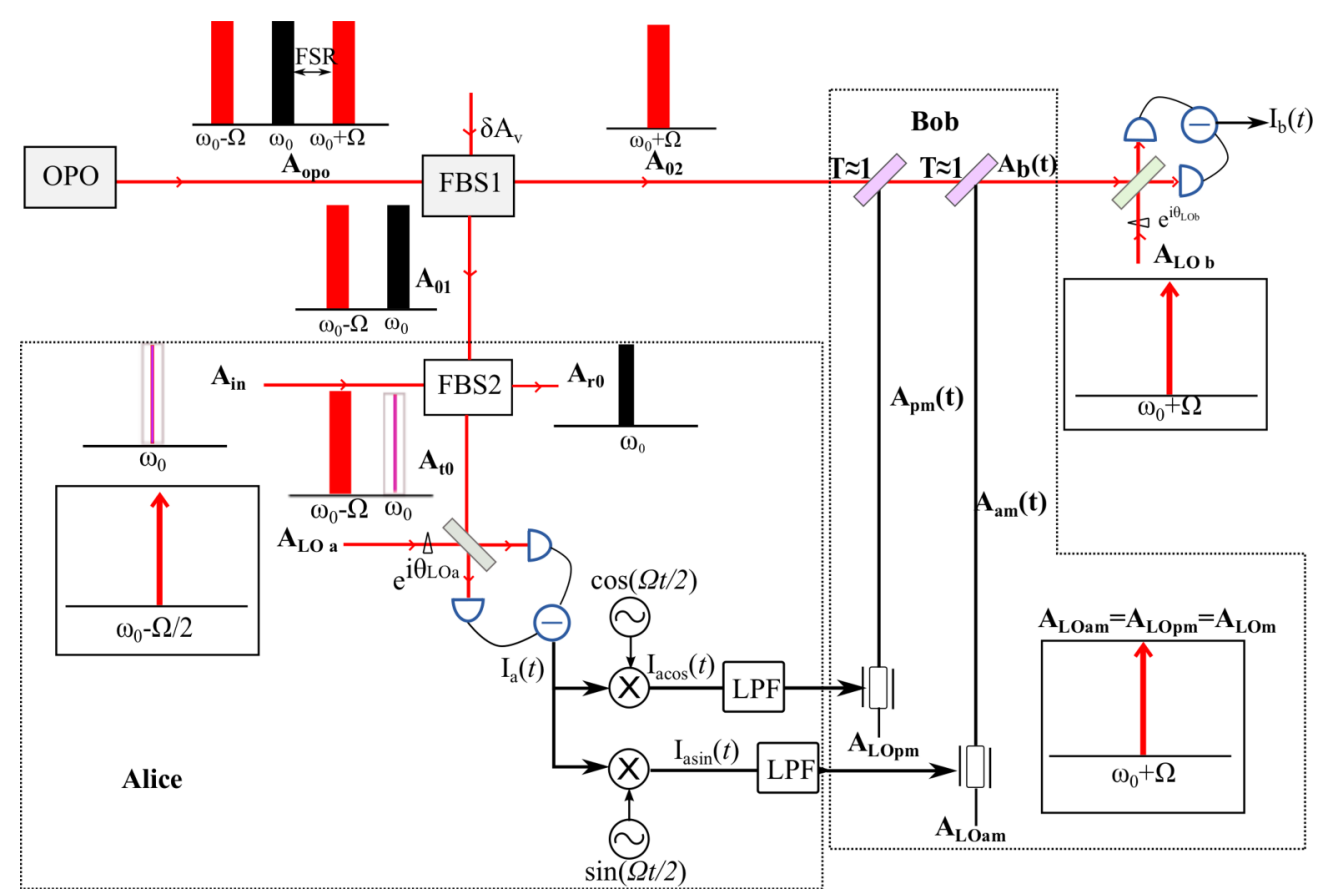

FIG. 4. (Color online) Schematic diagram of quantum teleportation using a pair of sidebands in a comb of entanglement.

On the other hand, FBS2 has a resonant frequency at $\omega_{0}-\Omega$. FBS2 combines the state to be teleported (appearing at $\omega_{0}$ ) with one of the output from FBS1 $\left(A_{01}\right)$. We obtain the field at "Alice", $A_{t 0}$, which is the combination of the state to be teleported, $A_{i n}$, and one of the entangled sidebands at $\omega_{0} \pm \Omega$. The field at Alice can be written as,

$$
\widetilde{A}_{t 0}(\omega)= \begin{cases}\widetilde{A}_{01}(\omega) & \omega_{0}-\Omega-\frac{\Delta \omega_{F B S_{2}}}{2}<\omega<\omega_{0}-\Omega+\frac{\Delta \omega_{F B S_{2}}}{2} \\ \widetilde{A}_{i n}(\omega) & \text { others. }\end{cases}
$$

Alice performs homodyne detection and demodulation with $\theta_{L O_{a}}=\frac{\pi}{2}, \omega_{L O_{a}}=\omega_{0}-\frac{\Omega}{2}$, and $\omega_{m_{a}}=\frac{\Omega}{2}$. Under those circumstances, we have

$\delta I_{a}(t)=i \bar{A}_{L O a} G_{\mathrm{det}}\left[e^{i\left(\omega_{0}-\Omega / 2\right) t} \delta A_{t 0}^{\dagger}(t)-e^{-i\left(\omega_{0}-\Omega / 2\right) t} \delta A_{t 0}(t)\right]$

After electronic demodulation with $\cos \left(\frac{\Omega t}{2}\right)$ and $\sin \left(\frac{\Omega t}{2}\right)$, we obtain,

$$
\begin{aligned}
\delta I_{\mathrm{acos}}(t)= & \frac{i \bar{A}_{L O a} G_{\mathrm{det}}}{2}\left[\left(e^{i \omega_{0} t}+e^{i\left(\omega_{0}-\Omega\right) t}\right) \delta A_{t 0}^{\dagger}(t)\right. \\
& \left.-\left(e^{-i\left(\omega_{0}-\Omega\right) t}+e^{-i \omega_{0} t}\right) \delta A_{t 0}(t)\right] \\
\delta I_{\mathrm{asin}}(t)= & \frac{\bar{A}_{L O a} G_{\mathrm{det}}}{2}\left[\left(e^{i \omega_{0} t}-e^{i\left(\omega_{0}-\Omega\right) t}\right) \delta A_{t 0}^{\dagger}(t)\right. \\
& \left.-\left(e^{-i\left(\omega_{0}-\Omega\right) t}-e^{-i \omega_{0} t}\right) \delta A_{t 0}(t)\right] .
\end{aligned}
$$

Alice sends these measurement results to "Bob". Then Bob displaces his field with those measurement results to reconstruct the input state. In order to perform displacement, Bob implements amplitude and phase modulation with $I_{\text {asin }}(t)$ and $I_{\text {acos }}(t)$ on frequency-shifted local oscillators, $A_{L O_{a m}}(t)=\left[\bar{A}_{L O_{m}}+\delta A_{L O_{a m}}(t)\right] e^{i\left(\omega_{0}+\Omega\right) t}$ and
$A_{L O_{p m}}(t)=\left[\bar{A}_{L O_{m}}+\delta A_{L O_{p m}}(t)\right] e^{i\left(\omega_{0}+\Omega\right) t}$. We have

$$
\begin{aligned}
\delta A_{a m}(t)= & \bar{A}_{L O_{m}} G_{a m} e^{i\left(\omega_{0}+\Omega\right) t} \delta I_{\mathrm{asin}}(t) \\
= & g_{a m}\left[\left(e^{i\left(2 \omega_{0}+\Omega\right) t}-e^{2 i \omega_{0} t}\right) \delta A_{t 0}^{\dagger}(t)\right. \\
& \left.-\left(e^{2 i \Omega t}-e^{i \Omega t}\right) \delta A_{t 0}(t)\right], \\
\delta A_{p m}(t)= & i \bar{A}_{L O_{m}} G_{p m} e^{i\left(\omega_{0}+\Omega\right) t} \delta I_{\mathrm{acos}}(t) \\
= & g_{p m}\left[\left(e^{2 i \Omega t}+e^{i \Omega t}\right) \delta A_{t 0}(t)\right. \\
& \left.-\left(e^{i\left(2 \omega_{0}+\Omega\right) t}+e^{2 i \omega_{0} t}\right) \delta A_{t 0}^{\dagger}(t)\right],
\end{aligned}
$$

where $G_{a m}=G_{p m}=G_{m}$ are gains of amplitude and phase modulations, and $g_{a m}=g_{p m}=\frac{\bar{A}_{L O_{m}} G_{\mathrm{det}} G_{m} \bar{A}_{L O_{a}}}{2}$. Correspondingly, in the frequency domain, we have

$$
\begin{aligned}
\delta \widetilde{A}_{a m}(\omega)= & g_{a m}\left[\delta \widetilde{A}_{t 0}\left(2 \omega_{0}+\Omega-\omega\right)^{\dagger}-\delta \widetilde{A}_{t 0}\left(2 \omega_{0}-\omega\right)^{\dagger}\right. \\
& \left.-\delta \widetilde{A}_{t 0}(\omega-2 \Omega)+\delta \widetilde{A}_{t 0}(\omega-\Omega)\right], \\
\delta \widetilde{A}_{p m}(\omega)= & g_{p m}\left[-\delta \widetilde{A}_{t 0}\left(2 \omega_{0}+\Omega-\omega\right)^{\dagger}-\delta \widetilde{A}_{t 0}\left(2 \omega_{0}-\omega\right)^{\dagger}\right. \\
& \left.+\delta \widetilde{A}_{t 0}(\omega-2 \Omega)+\delta \widetilde{A}_{t 0}(\omega-\Omega)\right] .
\end{aligned}
$$

After displacing $A_{02}$ by using these amplitude- and phasemodulated signals similar to Eq. (21) and setting $T^{2}=$ $2 R T g_{p m}=2 R g_{a m}=1$, we obtain the teleported state in 


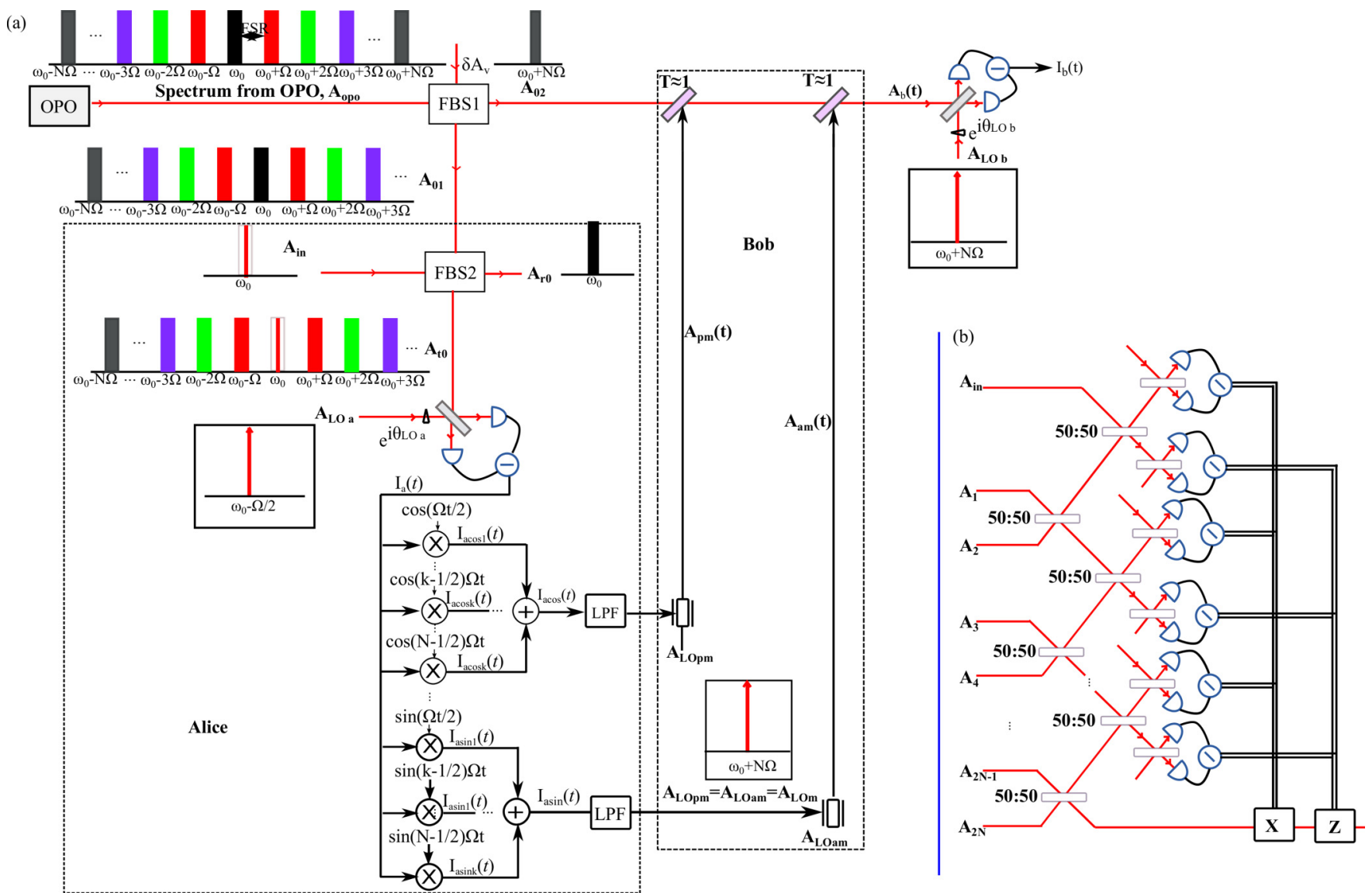

FIG. 5. (Color online) (a) Schematic diagram of sequential teleportation based on a comb of entanglement (b) Conventional sequential teleportation based on spatially separated $2 N$ squeezed vacuum states $\left(A_{1}\right.$ to $\left.A_{2 N}\right)$. X and $\mathrm{Z}$ are phase space displacements.

frequency domain as

$$
\delta \widetilde{A}_{b}(\omega)=\delta \widetilde{A}_{02}(\omega)+\delta \widetilde{A}_{t 0}(\omega-\Omega)-\delta \widetilde{A}_{t 0}\left(2 \omega_{0}-\omega\right)^{\dagger} .
$$

In order to verify this teleported state, we measure $\delta \widetilde{A}_{b}(\omega)$ with $\omega_{L O_{b}}=\omega_{0}+\Omega$,

$$
\begin{aligned}
\delta \widetilde{I}_{b, \theta_{L O b}}(\omega)= & \bar{A}_{L O b} G_{\operatorname{det}}\left[e^{-i \theta_{L O b}} \delta \widetilde{A}_{b}\left(\omega_{0}+\Omega+\omega\right)\right. \\
& \left.+e^{i \theta_{L O b}} \delta \widetilde{A}_{b}\left(\omega_{0}+\Omega-\omega\right)^{\dagger}\right] .
\end{aligned}
$$

If we focus on the DC term $(\omega=0)$, and set LO phase as $\theta_{L O_{b}}=0$ and $\frac{\pi}{2}$, we obtain

$$
\begin{aligned}
\delta \widetilde{I}_{b, 0}(\omega=0) & =\bar{A}_{L O b} G_{\operatorname{det}}\left[\delta \widetilde{A}_{b}\left(\omega_{0}+\Omega\right)+\delta \widetilde{A}_{b}\left(\omega_{0}+\Omega\right)^{\dagger}\right], \\
\delta \widetilde{I}_{b, \frac{\pi}{2}}(\omega=0) & =i \bar{A}_{L O b} G_{\operatorname{det}}\left[\delta \widetilde{A}_{b}\left(\omega_{0}+\Omega\right)^{\dagger}-\delta \widetilde{A}_{b}\left(\omega_{0}+\Omega\right)\right] .
\end{aligned}
$$

Substituting Eqs. (28), (29), (30), and (36) into Eq. (38), we have

$$
\begin{aligned}
\delta \widetilde{I}_{b, 0}(\omega=0)= & \bar{A}_{L O b} G_{\mathrm{det}}\left[\delta \tilde{X}_{\mathrm{opo}}\left(\omega_{0}+\Omega\right)+\delta \widetilde{X}_{i n}\left(\omega_{0}\right)\right. \\
& \left.-\delta \widetilde{X}_{\mathrm{opo}}\left(\omega_{0}-\Omega\right)\right], \\
\delta \widetilde{I}_{b, \frac{\pi}{2}}(\omega=0)= & \bar{A}_{L O b} G_{\mathrm{det}}\left[\delta \widetilde{Y}_{\mathrm{opo}}\left(\omega_{0}+\Omega\right)+\delta \widetilde{Y}_{i n}\left(\omega_{0}\right)\right. \\
& \left.+\delta \widetilde{Y}_{\mathrm{opo}}\left(\omega_{0}-\Omega\right)\right] .
\end{aligned}
$$

According to the correlation between $\omega_{0} \pm \Omega$ sidebands shown in Eq. (2), it can be seen that when the squeezing parameter $r$ goes to infinity $(r \rightarrow \infty)$, both the amplitude quadrature and phase quadrature of the input state are reconstructed at a different location and different frequency with unit fidelity. Since we obtain the teleported state at a different frequency, together with teleporting the state to a different location, we realize the teleportation in frequency space as well. Such a space-and-frequency teleportation scheme enables us to effectively link the frequency channels in FDM.

\section{V. $N$-SEQUENTIAL TELEPORTATION}

We generalize the quantum teleportation protocol in Sec. IV to a sequential teleportation scheme. We teleport an unknown input state in a frequency mode $\omega_{0}$ to a spatially separated frequency mode $\omega_{0}+N \Omega$ through $N$ bipartite entangled frequency modes ranging from $\omega_{0}-N \Omega$ to $\omega_{0}+N \Omega$, as shown in Fig. 5. We extend the number of modulation and demodulation operations shown in Fig. 4 from 2 to $2 N$ to take full advantage of all teeth in the comb. For comparison, Fig. 5(b) shows a setup for conventional sequential teleportation, where we need a number of beam splitters and homodyne detections [18]. 
By analogy with Eqs. (28) and (29), the outputs from FBS1 are

$$
\begin{aligned}
& \widetilde{A}_{01}(\omega)= \begin{cases}\delta \widetilde{A}_{v}(\omega) & \omega_{0}+N \Omega-\frac{\Delta \omega_{F B S_{1}}}{2}<\omega<\omega_{0}+N \Omega+\frac{\Delta \omega_{F B S_{1}}}{2} \\
\widetilde{A}_{\text {opo }}(\omega) & \text { others. }\end{cases} \\
& \widetilde{A}_{02}(\omega)= \begin{cases}\widetilde{A}_{\text {opo }}(\omega) & \omega_{0}+N \Omega-\frac{\Delta \omega_{F B S_{1}}}{2}<\omega<\omega_{0}+N \Omega+\frac{\Delta \omega_{F B S_{1}}}{2} \\
\delta \widetilde{A}_{v}(\omega) & \text { others. }\end{cases}
\end{aligned}
$$

For the output of FBS2, we obtain

$$
\delta \widetilde{A}_{t 0}(\omega)= \begin{cases}\delta \widetilde{A}_{i n}(\omega) & \omega_{0}-\frac{\Delta \omega_{F B S_{2}}}{2}<\omega<\omega_{0}+\frac{\Delta \omega_{F B S_{2}}}{2} \\ \delta \widetilde{A}_{01}(\omega) & \text { others }\end{cases}
$$

where $\Delta \omega_{B}<\Delta \omega_{F B S_{1,2}}<\Omega$. Alice measures this $\delta A_{t 0}$ with $\theta_{L O a}=\frac{\pi}{2}$ and $\omega_{L O_{a}}=\omega_{0}-\frac{\Omega}{2}$. After conducting a series of demodulations with $\cos \left(k-\frac{1}{2}\right) \Omega t$ and $\sin \left(k-\frac{1}{2}\right) \Omega t$ with $k=1,2, \ldots, N$ on $\delta I_{a}(t)$, we obtain

$$
\begin{gathered}
\delta I_{\text {acosk }}(t)=\frac{i \bar{A}_{L O a} G_{\mathrm{det}}}{2}\left[\left(e^{i\left[\omega_{0}+(k-1) \Omega\right] t}+e^{i\left(\omega_{0}-k \Omega\right) t}\right) \delta A_{t 0}^{\dagger}(t)-\left(e^{-i\left(\omega_{0}-k \Omega\right) t}+e^{-i\left[\omega_{0}+(k-1) \Omega\right] t}\right) \delta A_{t 0}(t)\right], \\
\delta I_{\text {asink }}(t)=\frac{\bar{A}_{L O a} G_{\mathrm{det}}}{2}\left[\left(e^{i\left[\omega_{0}+(k-1) \Omega\right] t}-e^{i\left(\omega_{0}-k \Omega\right) t}\right) \delta A_{t 0}^{\dagger}(t)-\left(e^{-i\left(\omega_{0}-k \Omega\right) t}-e^{-i\left[\omega_{0}+(k-1) \Omega\right] t}\right) \delta A_{t 0}(t)\right] .
\end{gathered}
$$

By electronically combining each of the $\delta I_{\text {acosk }}(t)$ and $\delta I_{\text {asink }}(t)$ for $k=1,2, \ldots, N$, we form $I_{\text {acos }}(t)$ and $I_{\text {asin }}(t)$, respectively. Those two signals are transmitted to Bob. Bob implements amplitude modulation and phase modulation with $I_{\text {asin }}(t)$ and $I_{\text {acos }}(t)$ on the frequency-shifted local oscillators $A_{L O_{a m}}(t)=\left[\bar{A}_{L O_{m}}+\delta A_{L O_{a m}}(t)\right] e^{i\left(\omega_{0}+N \Omega\right) t}$ and $A_{L O_{p m}}(t)=\left[\bar{A}_{L O_{m}}+\delta A_{L O_{p m}}(t)\right] e^{i\left(\omega_{0}+N \Omega\right) t}$. Bob then has

$$
\begin{aligned}
\delta A_{a m}(t) & =\bar{A}_{L O_{m}} G_{a m} e^{i\left(\omega_{0}+N \Omega\right) t} \sum_{k=1}^{N} \delta I_{\mathrm{asink}}(t) \\
& =g_{a m} \sum_{k=1}^{N}\left[\left(e^{i\left[2 \omega_{0}+(N+k-1) \Omega\right] t}-e^{i\left[2 \omega_{0}+(N-k) \Omega\right] t}\right) \delta A_{t 0}^{\dagger}(t)-\left(e^{i(N+k) \Omega t}-e^{i(N-k+1) \Omega t}\right) \delta A_{t 0}(t)\right], \\
\delta A_{p m}(t) & =i \bar{A}_{L O_{m}} G_{p m} e^{i\left(\omega_{0}+N \Omega\right) t} \sum_{k=1}^{N} \delta I_{\mathrm{acosk}}(t) \\
& =g_{p m} \sum_{k=1}^{N}\left[\left(e^{i(N+k) \Omega t}+e^{i(N-k+1) \Omega t}\right) \delta A_{t 0}(t)-\left(e^{i\left[2 \omega_{0}+(N+k-1) \Omega\right] t}+e^{i\left[2 \omega_{0}+(N-k) \Omega\right] t}\right) \delta A_{t 0}^{\dagger}(t)\right],
\end{aligned}
$$

where $G_{a m}=G_{p m}=G_{m}$ are gains of amplitude and phase modulations, and $g_{a m}=g_{p m}=\frac{\bar{A}_{L O_{m}} G_{\mathrm{det}} G_{m} \cdot \bar{A}_{L O_{a}}}{2}$. Correspondingly, in the frequency domain, we have

$$
\begin{aligned}
\delta \widetilde{A}_{a m}(\omega)= & g_{a m} \sum_{k=1}^{N}\left[\delta \widetilde{A}_{t 0}\left(2 \omega_{0}+(k+N-1) \Omega-\omega\right)^{\dagger}-\delta \widetilde{A}_{t 0}\left(2 \omega_{0}+(N-k) \Omega-\omega\right)^{\dagger}-\delta \widetilde{A}_{t 0}(\omega-(N+k) \Omega)\right. \\
& \left.+\delta \widetilde{A}_{t 0}(\omega-(N-k+1) \Omega)\right], \\
\delta \widetilde{A}_{p m}(\omega)= & g_{p m} \sum_{k=1}^{N}\left[-\delta \widetilde{A}_{t 0}\left(2 \omega_{0}+(k+N-1) \Omega-\omega\right)^{\dagger}-\delta \widetilde{A}_{t 0}\left(2 \omega_{0}+(N-k) \Omega-\omega\right)^{\dagger}+\delta \widetilde{A}_{t 0}(\omega-(N+k) \Omega)\right. \\
& \left.+\delta \widetilde{A}_{t 0}(\omega-(N-k+1) \Omega)\right] .
\end{aligned}
$$

After displacing $A_{02}$ by using these amplitude- and phase-modulated signals similar to Eq. (21) and setting $T^{2}=2 R T g_{p m}=$ $2 R g_{a m}=1$, we obtain the signal in frequency domain as

$$
\delta \widetilde{A}_{b}(\omega)=\sum_{k=1}^{N}\left[\delta \widetilde{A}_{02}(\omega)+\delta \widetilde{A}_{t 0}(\omega-(N-k+1) \Omega)-\delta \widetilde{A}_{t 0}\left(2 \omega_{0}+(N-k) \Omega-\omega\right)^{\dagger}\right] .
$$

In order to verify the teleported state, we measure $\delta A_{b}$ with $\omega_{L O b}=\omega_{0}+N \Omega$, then we obtain,

$$
\delta \widetilde{I}_{b, \theta_{L O b}}(\omega)=\bar{A}_{L O b} G_{\operatorname{det}}\left[e^{-i \theta_{L O b}} \delta \widetilde{A}_{b}\left(\omega_{0}+N \Omega+\omega\right)+e^{i \theta_{L O b}} \delta \widetilde{A}_{b}\left(\omega_{0}+N \Omega-\omega\right)^{\dagger}\right] .
$$


If we focus on the DC term $(\omega=0)$, and set LO phase as $\theta_{L O_{b}}=0$ and $\frac{\pi}{2}$, we obtain

$$
\begin{aligned}
\delta \widetilde{I}_{b, 0}(\omega=0) & =\bar{A}_{L O b} G_{\mathrm{det}}\left[\delta \widetilde{A}_{b}\left(\omega_{0}+N \Omega\right)+\delta \widetilde{A}_{b}\left(\omega_{0}+N \Omega\right)^{\dagger}\right], \\
\delta \widetilde{I}_{b, \frac{\pi}{2}}(\omega=0) & =i \bar{A}_{L O b} G_{\mathrm{det}}\left[\delta \widetilde{A}_{b}\left(\omega_{0}+N \Omega\right)^{\dagger}-\delta \widetilde{A}_{b}\left(\omega_{0}+N \Omega\right)\right] .
\end{aligned}
$$

Substituting Eq. (47) into Eq. (49), we have

$$
\begin{gathered}
\delta \widetilde{I}_{b, 0}(\omega=0)=\bar{A}_{L O b} G_{\text {det }}\left[\delta \widetilde{X}_{02}\left(\omega_{0}+N \Omega\right)+\sum_{k=1}^{N}\left(\delta \widetilde{X}_{t 0}\left(\omega_{0}+(k-1) \Omega\right)-\delta \widetilde{X}_{t 0}\left(\omega_{0}-k \Omega\right)\right)\right], \\
\delta \widetilde{I}_{b, \frac{\pi}{2}}(\omega=0)=\bar{A}_{L O b} G_{\text {det }}\left[\delta \widetilde{Y}_{02}\left(\omega_{0}+N \Omega\right)+\sum_{k=1}^{N}\left(\delta \widetilde{Y}_{t 0}\left(\omega_{0}+(k-1) \Omega\right)+\delta \widetilde{Y}_{t 0}\left(\omega_{0}-k \Omega\right)\right)\right] .
\end{gathered}
$$

Based on Eqs. (40), (41), (42), we can rewrite Eq. (50) as,

$$
\begin{aligned}
& \delta \widetilde{I}_{b, 0}(\omega=0)=\bar{A}_{L O b} G_{\mathrm{det}}\left[\delta \widetilde{X}_{i n}\left(\omega_{0}\right)+\sum_{k=1}^{N}\left(\delta \widetilde{X}_{\mathrm{opo}}\left(\omega_{0}+k \Omega\right)-\delta \widetilde{X}_{\mathrm{opo}}\left(\omega_{0}-k \Omega\right)\right)\right], \\
& \delta \widetilde{I}_{b, \frac{\pi}{2}}(\omega=0)=\bar{A}_{L O b} G_{\mathrm{det}}\left[\delta \widetilde{Y}_{i n}\left(\omega_{0}\right)+\sum_{k=1}^{N}\left(\delta \widetilde{Y}_{\mathrm{opo}}\left(\omega_{0}+k \Omega\right)+\delta \widetilde{Y}_{\mathrm{opo}}\left(\omega_{0}-k \Omega\right)\right)\right] .
\end{aligned}
$$

Since the sidebands from the output of an OPO have correlations, as shown in Eq. (2), the teleported state is identical to the input in the limit of $r \rightarrow \infty$. In a case of finite squeezing parameter $r$, each teleportation step adds extra noise. Thus the teleported state has accumulated noise proportional to the number of teleportation steps. It is noted that the accumulation of noise per teleportation step is no greater than that in the traditional approach to sequential teleportation [18].

In this sequential teleportation protocol, the teleportation steps occur simultaneously in time but sequentially in frequency. Although quantum teleportation is in principle an identity operation on the input state, it can be extended to measurement-based QC, where unitary operations are realized by changing the measurement bases [3,5]. For example, if we add a frequency-dependent phase shifter in front of Alice's detector, then we may change the measurement bases appropriately, and may realize a series of Gaussian operations via sequential teleportation. Efficient ways to apply our protocol to measurement-based QC deserves further study, and may stimulate development of practical FDM quantum information protocols.

\section{CONCLUSIONS}

We proposed protocols for entanglement swapping and sequential quantum teleportation using a comb of entanglement and wide-band frequency-resolved homodyne detection. A comb of entanglement generated from a subthreshold cw OPO inherently possesses EPR-like correlations. Electronic demodulation following the wide-band homodyne detector enables us to directly access the sum and/or difference of quadratures between different frequency teeth without the need for optical mixing. These measurements produce EPR nullifiers and thus can be used as the basis for teleportation protocols. Our protocol for sequential space-and-frequency teleportation effectively links arbitrary frequency channels for frequencydivision multiplexing, and has applications in universal quantum computation and large-capacity quantum communication.

\section{ACKNOWLEDGMENT}

This work was supported financially by the Australian Research Council, Grant No. CE110001029 and the Deutsche Forschungsgemeinschaft through the Centre for Quantum Engineering and Space-Time Research (QUEST).
[1] D. Gottesman and I. L. Chuang, Nature (London) 402, 390 (1999).

[2] R. Raussendorf and H. J. Briegel, Phys. Rev. Lett. 86, 5188 (2001).

[3] N. C. Menicucci, P. van Loock, M. Gu, C. Weedbrook, T. C. Ralph, and M. A. Nielsen, Phys. Rev. Lett. 97, 110501 (2006).

[4] N. C. Menicucci, Phys. Rev. Lett. 112, 120504 (2014).

[5] S. Yokoyama, R. Ukai, S. C. Armstrong, C. Sornphiphatphong, T. Kaji, S. Suzuki, J. Yoshikawa, H. Yonezawa, N. C. Menicucci, and A. Furusawa, Nature Photon. 7, 982 (2013).

[6] M. Pysher, Y. Miwa, R. Shahrokhshahi, R. Bloomer, and O. Pfister, Phys. Rev. Lett. 107, 030505 (2011).
[7] M. Chen, N. C. Menicucci, and O. Pfister, Phys. Rev. Lett. 112, 120505 (2014).

[8] O. Pinel, P. Jian, R. M. de Araújo, J. Feng, B. Chalopin, C. Fabre, and N. Treps, Phys. Rev. Lett. 108, 083601 (2012).

[9] J. Roslund, R. M. de Araújo, S. Jiang, C. Fabre, and N. Treps, Nature Photon. 8, 109 (2014).

[10] M. Heurs, J. G. Webb, A. E. Dunlop, C. C. Harb, T. C. Ralph, and E. H. Huntington, Phys. Rev. A 81, 032325 (2010).

[11] K. Kuntz, H. Song, J. Webb, T. Wheatley, A. Furusawa, T. Ralph, and E. Huntington, in CLEO: 2014 (Optical Society of America, Washington, DC, 2014), p. FTh3A.3. 
[12] M. Gu, C. Weedbrook, N. C. Menicucci, T. C. Ralph, and P. van Loock, Phys. Rev. A 79, 062318 (2009).

[13] D. F. Wall and G. Milburn, Quantum Optics (Springer-Verlag, Berlin, 1995).

[14] J. Zhang, Phys. Rev. A 67, 054302 (2003).

[15] N. C. Menicucci, S. T. Flammia, and P. van Loock, Phys. Rev. A 83, 042335 (2011).
[16] E. H. Huntington, G. N. Milford, C. Robilliard, T. C. Ralph, O. Glöckl, U. L. Andersen, S. Lorenz, and G. Leuchs, Phys. Rev. A 71, 041802 (2005).

[17] N. Takei, H. Yonezawa, T. Aoki, and A. Furusawa, Phys. Rev. Lett. 94, 220502 (2005).

[18] H. Yonezawa, A. Furusawa, and P. van Loock, Phys. Rev. A 76, 032305 (2007). 